\title{
SLOW-WAVE ELECTRODE STRUCTURES FOR THE ESS 2.5 MeV FAST CHOPPER
}

\author{
Michael A. Clarke-Gayther, CLRC RAL, Didcot, United Kingdom.
}

\begin{abstract}
Slow-wave electrode designs for the European Spallation Source (ESS) $2.5 \mathrm{MeV}$ fast beam chopper are described. Broad-band transmission line properties for the initial planar and helical designs have been refined by modelling electromagnetic fields in the Finite Difference Time Domain (FDTD) 'Microwave Studio' code. A compact, radiation hard, vacuum compatible helical structure made from linked strip-line and micro-strip sections has been developed. Simulated characteristics in the frequency and time domain, and the effects of E-field non-uniformity are presented.
\end{abstract}

\section{INTRODUCTION}

The next generation of high power proton drivers for pulsed neutron sources (ESS) [1] and neutrino factories [2], call for significant technical development in many areas of accelerator design. The ESS $\mathrm{H}^{-}$linac fast beam choppers in the $2.5 \mathrm{MeV}$ medium energy transport (MEBT) lines, must produce the precisely defined gaps in the $280 \mathrm{MHz}$ bunched beam that enable low beam loss operation during accumulator ring injection and extraction. Stringent beam loss requirements in the downstream linacs and rings dictate that the chopping fields should rise and fall within the beam bunch interval of $2.9 \mathrm{~ns}$, to eliminate the possibility of acceleration, and subsequent loss of partially chopped beam. Slow-wave (E-field) transmission line structures have demonstrated field transition times in the nanosecond regime [3,4], and ESS chopping schemes utilising these structures have been identified [5] and refined [6]. They are designed with the aid of 3D high frequency field modelling codes, where the complex geometry, extended electrical length, and the effects of inter-electrode coupling set a practical limit on the computational accuracy of the broad-band properties. Speed and/or accuracy of computation, have been enhanced by identifying small repetitive structures and modelling their properties in the finite element frequency domain (FEFD) HFSS code, and more recently, in the finite difference time domain (FDTD) 'Microwave Studio' code. Time domain characteristics for the complete structures were analysed in a high frequency SPICE based circuit simulator where the complete structures were modelled by linking the repetitive twoport modules in large series arrays.

\section{SLOW-WAVE ELECTRODE DESIGN}

The basic features and function of the proposed slowwave electrode structures are shown in Figure 1, where partial chopping of beam bunches is avoided by ensuring that the deflecting E-field propagates at the beam bunch velocity.

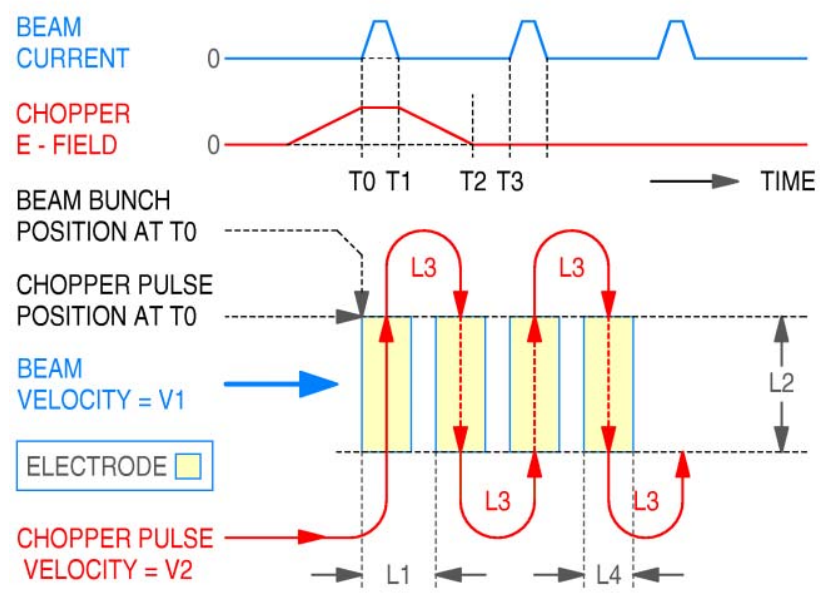

Figure 1: Slow-wave electrode design

In Figure 1:

L2 is the transverse extent of the beam.

$\mathrm{T}(\mathrm{L} 1)$ is the beam transit time for distance $\mathrm{L} 1$.

$\mathrm{T}(\mathrm{L} 2)$ is the pulse transit time in vacuum for distance $\mathrm{L} 2$.

$\mathrm{T}(\mathrm{L} 3)$ is the pulse transit time in dielectric media for distance L3.

L4 is the electrode width.

For the generalised slow wave structure:

Maximum value for $\mathrm{L} 1=\mathrm{V} 1(\mathrm{~T} 3-\mathrm{T} 1) / 2$

Minimum Value for $\mathrm{L} 1=\mathrm{L} 2(\mathrm{~V} 1 / \mathrm{V} 2)$

$\mathrm{T}(\mathrm{L} 1)=\mathrm{L} 1 / \mathrm{V} 1=\mathrm{T}(\mathrm{L} 2)+\mathrm{T}(\mathrm{L} 3)$

The relationships for field (E), and transverse displacement ( $\mathrm{x})$, where $\mathrm{q}$ is the electronic charge, $\mathrm{v}$ is the beam velocity, $\mathrm{m}_{0}$ is the rest mass, $\mathrm{z}$ is the effective electrode length, $\theta$ is the required deflection angle, $\mathrm{V}$ is the deflecting potential, and $\mathrm{d}$ is the electrode gap, are:

$$
E=\tan \theta \cdot m_{0} \cdot \frac{v^{2}}{q \cdot z}, \quad E=\frac{V}{d}, \quad x=\frac{q \cdot E \cdot z^{2}}{2 \cdot m_{0} \cdot v^{2}}
$$

Inspection shows that for given values of $m_{0}, v$, and $V$, large $\theta$ and $\mathrm{x}$ are obtained when $\mathrm{z}$ is large and $\mathrm{d}$ is small. The inter-electrode gap shown as L1-L4 in Figure 1, must be made significant if pulse distortion due to interelectrode coupling is to be minimised.

For a given overall structure length, the effective length (z) will therefore, be maximised, by maximising the electrode width (L4). 


\section{ELECTRODE STRUCTURES}

Planar slow-wave structure A, helical structure B, and the procedures adopted in their design, have been previously described [7]. Key parameters, common to those designs and to the new helical structure $\mathrm{C}$, are listed in Table 1.

Table 1: Slow-wave structure parameters

\begin{tabular}{|l|c|c|c|}
\hline Beam velocity & $\mathrm{v}$ & $2.184 \mathrm{e} 7$ & $\mathrm{~m} . \mathrm{s}^{-1}$ \\
\hline Mechanical length & & 400.0 & $\mathrm{~mm}$ \\
\hline Electrode to beam axis gap & $\mathrm{d}$ & 6.0 & $\mathrm{~mm}$ \\
\hline Beam aperture & & 11.0 & $\mathrm{~mm}$ \\
\hline Deflection angle & $\theta$ & 16 & $\mathrm{mr}$ \\
\hline Deflection potential $( \pm)$ & $\mathrm{Z}$ & 1.6 & $\mathrm{kV}$ \\
\hline Characteristic impedance & & $50 \pm 0.5$ & $\Omega$ \\
\hline Total structure delay & & 18.04 & $\mathrm{~ns}$ \\
\hline No. of sections & $\mathrm{T}(\mathrm{L} 1)$ & 0.87 & $\mathrm{~ns}$ \\
\hline Section delay & & 2.9 & $\mathrm{~ns}$ \\
\hline Pulse transition time (1-90,90-1\%) & & $0-500$ & $\mathrm{MHz}$ \\
\hline Structure bandwidth & $\mathrm{L} 1$ & 19.0 & $\mathrm{~mm}$ \\
\hline Section pitch & $\mathrm{L} 4$ & 14 & $\mathrm{~mm}$ \\
\hline Strip-line width & & 0.45 & $\mathrm{~mm}$ \\
\hline Strip-line thickness & & &
\end{tabular}

\section{Helical electrode structure $C$}

A mechanical schematic of structure $\mathrm{C}$ is shown in Figure 2. The compact helical structure is formed by strip-line sections near the beam axis, linked by sections of micro-strip. Micro-strip to strip-line transitions, and ceramic strip-line supports, are capacitively compensated by structure and notch dimensioning, respectively.

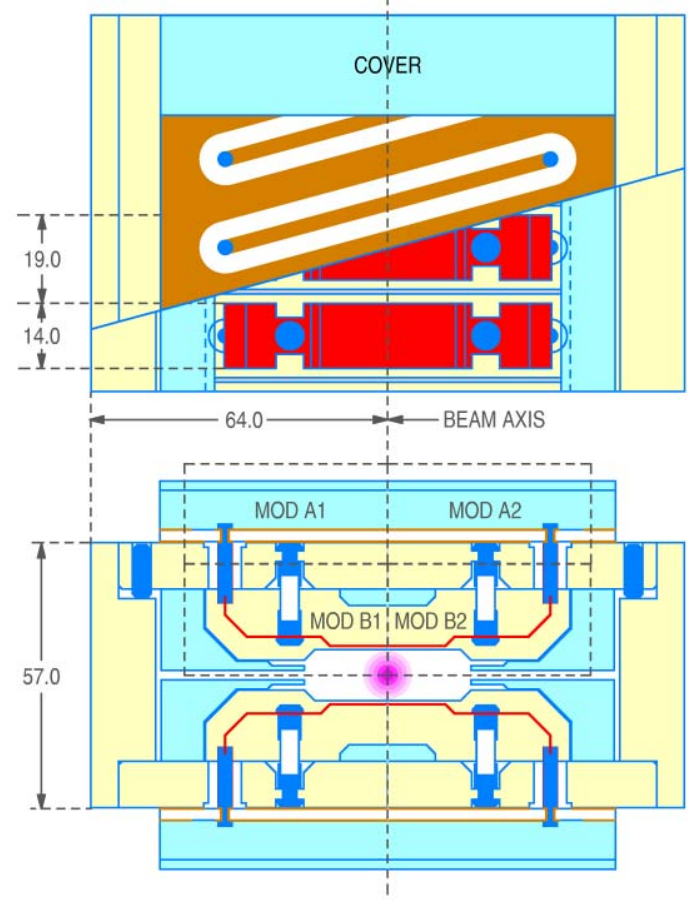

KEY: $\square$ STRIP-LINE $\square \square$ BODY $\square$ CERAMIC SCALE: mm $\square$ MICROSTRIP (70 um) / SUBSTRATE (2.54 mm / Er = 6)

Figure 2: Electrode structure C
Finite element models for two modules, identified as MOD A1/A2 and B1/B2 in Figure 2, were analysed. Simulated S-parameters for the modules are shown in Figure 3.

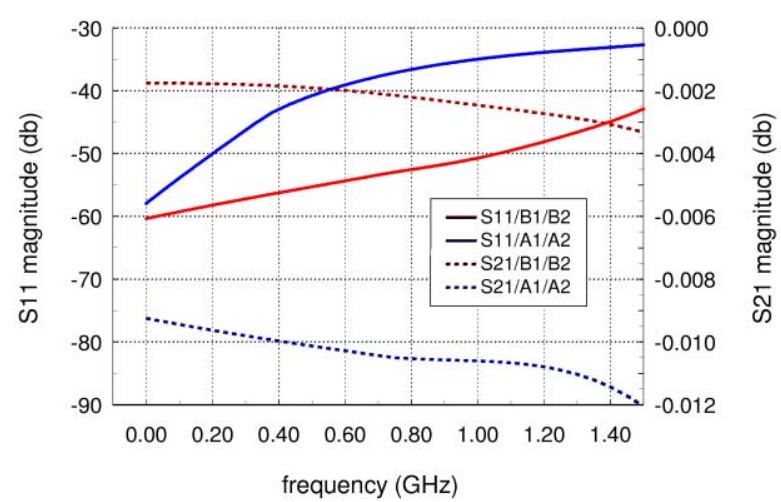

Figure 3: Structure C / Module S-Parameters

Time and frequency domain characteristics for the complete structure were computed by circuit simulation of series connected, two port modules, and lumped element inter-electrode, coupling capacitors, as shown in Figure 4.

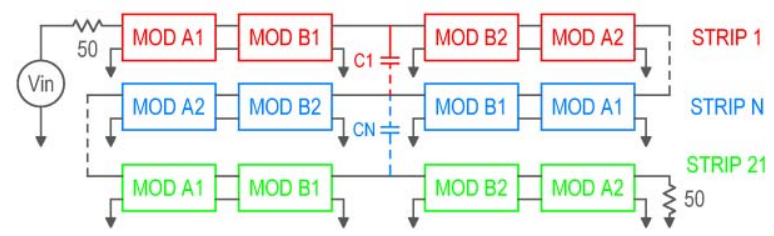

Figure 4: Structure C / Spice model

Simulated reflection and transmission characteristics in the frequency domain are shown in Figure 5. These indicate that the resonant frequencies of the structure are safely above the upper limit of the required bandwidth.

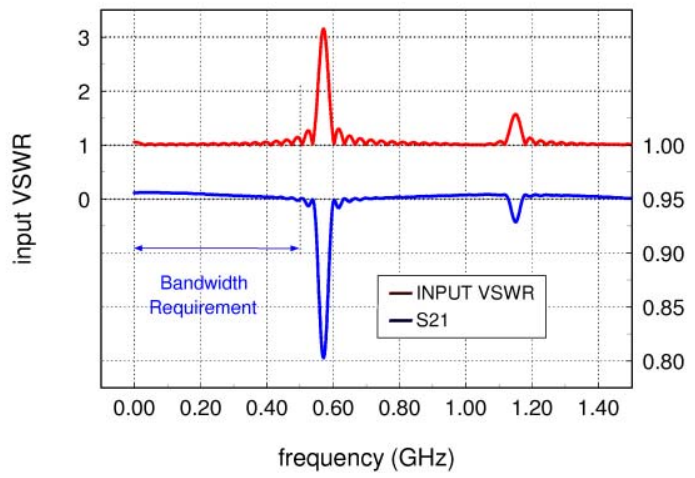

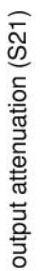

Figure 5: Structure C / Frequency Domain

Simulated pulse transmission characteristics in the time domain are shown in Figure 6. These indicate that structure $\mathrm{C}$ may exhibit a higher transmission loss and more pulse shape distortion than structures A or B. 


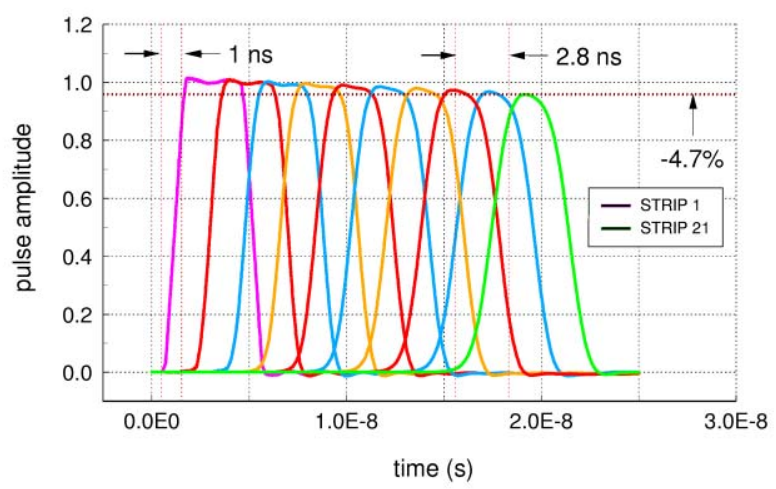

Figure 6: Structure C / Time Domain

\section{E-FIELD UNIFORMITY}

Uniformity of the beam-deflecting component of the simulated $\mathrm{E}$ field has been analysed. The variation in magnitude of this component as a function of axial position, as shown in Figure 7, exhibits the expected periodicity of the structure. This analysis predicts an average or rms field of $\sim 73 \%$ of the equivalent infinite parallel plate field level.

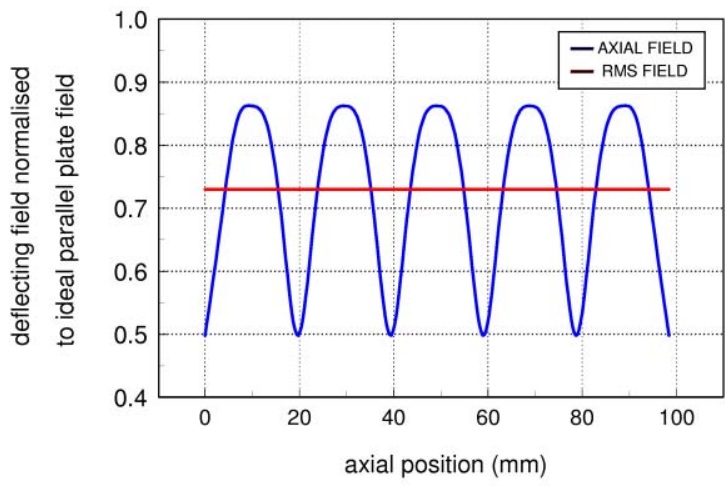

Figure 7: Structure C / Axial field distribution

Variation in the magnitude of the beam-deflecting component of the simulated $\mathrm{E}$ field, as a function of transverse position, as shown in Figure 8, indicates that structures $\mathrm{A}$ and $\mathrm{C}$ exhibit better field uniformity in this plane, than structure B.

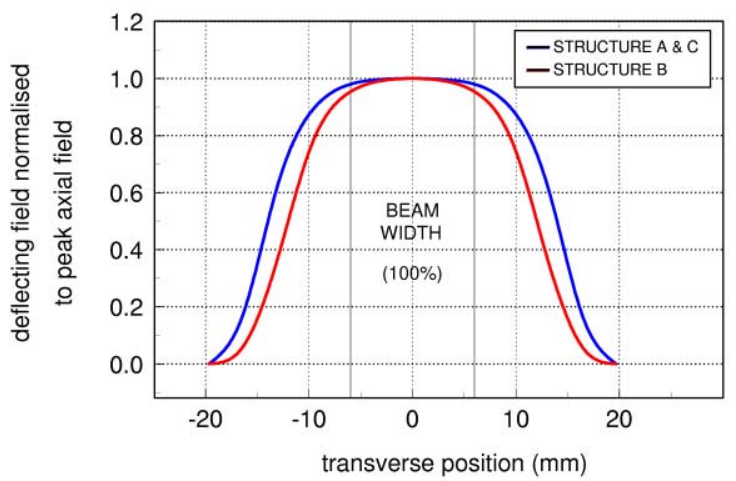

Figure 8: Structure C / Transverse field distribution

\section{SUMMARY}

Three slow wave structures for the ESS $2.5 \mathrm{MeV}$ beam chopper have been modelled using finite element and circuit simulation codes. Planar slow-wave structure A, helical structure B, and the procedures adopted in their design, have been previously described [7]. An important common feature of those structures, and of the new helical structure $\mathrm{C}$, is the radiation hard, vacuum compatible, strip-line configuration near the beam axis. Dispersion in the frequency domain, and the resultant pulse distortion due to inter-electrode coupling, have been minimised in these designs, by the introduction of extended inter-electrode ground planes.

Simulated frequency and time domain characteristics indicate that all three of the candidate structures may meet the required ESS specifications for chopper pulse fidelity.

Helical structure $\mathrm{C}$ was developed to address the need for a compact structure (more compact than helical structure B), but with the superior transverse field uniformity of planar structure A. These two goals have been achieved, at the expense of a small increase in transmission loss and pulse distortion. Last but not least, the mechanical construction of structure $\mathrm{C}$ may well turn out to be the least demanding of the three candidate structures.

\section{REFERENCES}

[1] The ESS Accelerator and Beam Lines with the 280/560 MHz Linac, Report No. ESS-01-1A, December 2001.

[2] C. R. Prior, and G. H Rees, 'RAL Proton Driver Studies for a Neutrino Factory', Proceedings of the Neutrino Factory Workshop, NUFACT'00, Monterey, California, June 2000.

[3] S. S. Kurennoy, and J. F. Power, 'Development of Meander-Line current structure for SNS fast $2.5 \mathrm{MeV}$ Beam Chopper', Proc. of the 7th European Particle Accelerator Conference, Vienna, Austria, June 2000, p.336-338.

[4] F. Caspers, A. Mostacci, S. Kurennoy, 'Fast Chopper Structure for the CERN SPL', Proc. of the eighth European Particle Accelerator Conference, Paris, France, 3-7 June, 2002, p. 873-875.

[5] M. A. Clarke-Gayther, 'Modulator Systems for the ESS 2.5 MeV Fast Chopper', Proc. of the 2001 Particle Accelerator Conference, Chicago, USA, p. 4062-4065.

[6] M. A. Clarke-Gayther, 'A Fast Chopper for the ESS 2.5 MeV Beam Transport Line', Proc. of the eighth European Particle Accelerator Conference, Paris, France, 3-7 June, 2002, p. 2136-2138.

[7] M. A. Clarke-Gayther, Yi Nong Rao, Dag Reistad, 'Slow-wave electrode structures for the ESS $2.5 \mathrm{MeV}$ Fast Chopper', Proc. of the eighth European Particle Accelerator Conference, Paris, France, 3-7 June, 2002, p. 2133-2135. 